\title{
Rapid and Low-Cost Culture-Based Method for Diagnosis of Mucormycosis Using a Mouse Model
}

\author{
Afsane Vaezi1,2, Hamed Fakhim ${ }^{3,4}$, Macit Ilkit ${ }^{5}$, Leila Faeli2,6, Mahdi Fakhar7, \\ Vahid Alinejad $^{8}$, Nathan P. Wiederhold ${ }^{9}$ and Hamid Badali ${ }^{1,2,9 *}$
}

${ }^{1}$ Invasive Fungi Research Center, School of Medicine, Mazandaran University of Medical Sciences, Sari, Iran, ${ }^{2}$ Department of Medical Mycology, School of Medicine, Mazandaran University of Medical Sciences, Sari, Iran, ${ }^{3}$ Department of Medical Parasitology and Mycology, Faculty of Medicine, Urmia University of Medical Sciences, Urmia, Iran, ${ }^{4}$ Infectious Diseases and Tropical Medicine Research Center, Isfahan University of Medical Sciences, Isfahan, Iran, ${ }^{5}$ Division of Mycology, Department of Microbiology, Faculty of Medicine, University of Çukurova, Adana, Turkey, ${ }^{6}$ Student Research Committee, Mazandaran University of Medical Sciences, Sari, Iran, ${ }^{7}$ Toxoplasmosis Research Center, Department of Parasitology, Mazandaran University of Medical Sciences, Sari, Iran, ${ }^{8}$ Patient Safety Research Center, Urmia University of Medical Sciences, Urmia, Iran, ${ }^{9}$ Fungus Testing Laboratory, Department of Pathology and Laboratory Medicine, University of Texas Health Science Center at San Antonio, San Antonio, TX, United States

Prompt and targeted antifungal treatment has a positive impact on the clinical outcome of mucormycosis; however, current diagnostic tools used in histopathology laboratories often fail to provide rapid results. Rapid culture-based strategies for early diagnosis of Mucorales infections, which may influence treatment decisions, are urgently needed. Herein, we evaluated a microculture assay for the early diagnosis of mucormycosis in an immunocompetent murine model of disseminated infection, by comparing it with traditional diagnostic methods. The assay specificity was assessed using blood $(n=90)$ and tissue $(n=90)$ specimens obtained from mice infected with Rhizopus arrhizus using different inoculum sizes $\left[1 \times 10^{4}, 1 \times 10^{5}\right.$, and $1 \times 10^{6}$ colony forming units (CFUs)/mouse] and blood $(n=15)$ and tissue specimens $(n=15)$ from uninfected mice. Surprisingly, 26 of 90 (28.9\%) blood samples revealed positive results by microculture, whereas all blood samples were negative when assayed by conventional culture. The overall positive conventional culture rate for the mouse tissue (kidney) samples was $31.1 \%$ (28/90). The calculated sensitivity for kidney microculture was 98.8\% [95\% confidence interval (Cl) 96.6-100], with an assay specificity of 100\%. Hence, the microculture assay may be useful for rapid culturing and diagnosis of mucormycosis caused by $R$. arrhizus directly in blood and tissue samples. Hence, this method may allow for the timely administration of an appropriate treatment.

Keywords: microculture, rapid diagnosis, Rhizopus arrhizus, mucormycosis, murine model

\section{INTRODUCTION}

Mucormycosis (previously called zygomycosis), an aggressive infection caused by mucoralean fungi, is the third most prevalent fungal disease after candidiasis and aspergillosis, among populations at high risk, including those with uncontrolled diabetes, solid organ or allogeneic stem cell transplant recipients, and patients undergoing immunosuppressive therapies (Petrikkos et al., 2012; Danion et al., 2015; Farmakiotis and Kontoyiannis, 2016; Hoenigl et al., 2018; Cornely et al., 2019). The reported incidence of mucormycosis is $0.2-95$ cases per $1,000,000$ individuals in 
Europe; 3.0 cases per 1,000,000 individuals in the United States; 1.2 cases per 1,000,000 individuals in Canada; and 0.6 cases per $1,000,000$ individuals in Australia (Skiada et al., 2012). Although few studies in Asia have reported the prevalence of Mucorales infections (Yamazaki et al., 1999; Chakrabarti and Singh, 2014; Vaezi et al., 2016), a national investigation of medical autopsies revealed that the incidence of these infections in Japan increased by $16 \%$ in a 20 -year span (Prakash and Chakrabarti, 2019). Early diagnosis and application of multimodal treatment, including appropriate antifungal therapy, may have a positive impact on clinical outcomes in patients with mucormycosis, including improved survival rates (Spellberg et al., 2005; Skiada et al., 2018).

The current gold standard for diagnosing mucormycosis is based on histopathological and mycological findings, followed by unspecific radiological criteria. However, these procedures require specialized expertise and the results are often not available in a timely fashion (Hammond et al., 2011; Cornely et al., 2019). To enhance outcomes, patients suspected to have mucormycosis should be immediately treated. Despite our improved understanding of the disease and the availability of various medico-surgical treatments, the survival rate in mucormycosis patients remains poor (Kontoyiannis and Lewis, 2011; Katragkou et al., 2014; Pilmis et al., 2018; Skiada et al., 2018). Therefore, there is a need for novel diagnostic assays. Several new molecular methods for the diagnosis of mucormycosis have been reported (Hammond et al., 2011; Dadwal and Kontoyiannis, 2018); however, these techniques may lack sensitivity, can be time-consuming and expensive to perform, and are not universally available (Katragkou et al., 2014).

The choice of an effective treatment regimen against mucormycosis requires early diagnosis and identification of the causative pathogen and its antifungal susceptibility profile, for which a positive culture is needed (Walsh et al., 2014; Hoenigl et al., 2018; Cornely et al., 2019). However, due to the unique physiology of these etiological agents (e.g., fragile and non-septate hyphae), cultures are frequently negative, and the processing of clinical specimens requires a suspicion of Mucorales as the causative agent and experienced laboratory personnel than may be required for fungi with septate hyphae (Ben-Ami et al., 2009; Hammond et al., 2011; Lewis et al., 2013; Cornely et al., 2019).

Here, we report the first study to evaluate a microculture assay as a putative, rapid, and low-cost culture-based method for the early diagnosis of mucormycosis. An established murine model was utilized to compare the performance of this microculture assay with those of traditional diagnostic methods.

\section{MATERIALS AND METHODS}

\section{Isolate and Inocula}

Rhizopus arrhizus var. arrhizus clinical isolate (CBS 112.07), obtained from the reference culture collection of the Westerdijk Fungal Biodiversity Institute (Utrecht, Netherlands), was used in this study. Species identity was confirmed by DNA sequence analysis of the internal transcribed spacer (ITS) region of ribosomal DNA (rDNA), as previously described (Nagao et al., 2005). For inoculum preparation, the strain was sub-cultured onto potato dextrose agar (PDA) at $37^{\circ} \mathrm{C}$ (Difco, Leeuwarden, Netherlands) 10 days before the inoculation in mice to ensure viability and purity. On the day of inoculation, sterile phosphatebuffered saline (PBS) containing $0.1 \%$ (v/v) Tween 20 was added to the plate, and the surface of colonies was gently scraped. After centrifugation at $15,000 \mathrm{rpm}$ for $15 \mathrm{~min}$, the supernatant was removed, and the cells were washed twice in PBS. The spore count was enumerated with a hemocytometer for preparing the final inocula. The cell concentrations were adjusted to three different inoculum sizes, $1 \times 10^{4}, 1 \times 10^{5}$, and $1 \times 10^{6}$ spores $/ \mathrm{ml}$. To confirm each inoculum size, dilutions were prepared and streaked onto PDA, and the fungal colonies were enumerated after $24 \mathrm{~h}$ of incubation at $30^{\circ} \mathrm{C}$.

\section{Animal Model}

Female immunocompetent ICR mice (weighing 22-25 g, 6week old, $n=105$ ) were purchased from the Royan Institute (Tehran, Iran). The animals were housed in groups of 30 mice each at the Animal Experimentation Facility under standard conditions. All mice were provided food and water ad libitum and were monitored daily, based on the recommendations of the guide for the Care and Use of Laboratory Animals of the National Research Council (2011). All animal experiments were approved by the Institutional Animal Ethical Committee (IAEC) of Mazandaran University of Medical Sciences, Sari, Iran (IR.MAZUMS.REC.1397.9).

\section{Experimental Model of Disseminated Infection}

In total, 90 mice were randomly divided into three groups $(n=30$ per group), and a $0.2 \mathrm{ml}$ solution containing one of the three inocula $\left[1 \times 10^{4}, 1 \times 10^{5}\right.$, and $1 \times 10^{6}$ colony forming units (CFUs)/mouse] was injected into the lateral tail vein of each mouse. Pilot experiments demonstrated that the inoculum sizes of $1 \times 10^{4}, 1 \times 10^{5}$, and $1 \times 10^{6} \mathrm{CFU} / \mathrm{mouse}$ proved to be the optimal doses leading to a severe infection; all animals died within 10 days of infection. In the fungal burden arm, mice were sacrificed by cervical dislocation on experimental day 4 postinfection. After sacrifice, kidneys were removed, homogenized, serially diluted (1:10), and plated on Sabouraud dextrose agar (SDA) for CFU/g calculation. The control group $(n=15)$ received intravenous injections of cell-free PBS using the same method. Mice were assessed at least twice daily, and moribund mice were euthanized by exsanguination (intracardiac puncture under general anesthesia) (Conti et al., 2014) after detecting symptoms of disseminated infection. Moribund animals were identified by the following criteria: decreased activity, inability to eat or drink, hypothermia, hunched posture, and torticollis or barrel rolling.

\section{Histopathological and Mycological Characterization}

Blood and tissue (kidney) samples were recovered under aseptic conditions. Blood samples were collected by cardiac puncture 
(approximately $200 \mu \mathrm{l}$ into heparin-coated tubes) and were stored at $-20^{\circ} \mathrm{C}$ until further analysis. The kidneys were minced and used for histopathology, microculture assay, traditional culture analysis. Kidney samples were first fixed in $10 \%$ (w/v) buffered formalin, dehydrated, paraffin-embedded (FFPE), sectioned (5- $\mu \mathrm{m}$-thick sections), and stained with Periodic acidSchiff (PAS) for direct microscopic examination, as previously described (Rickerts, 2016). Minced kidney and blood samples were also cultured on SDA and brain heart infusion (BHI) agar at $35^{\circ} \mathrm{C}$. Moreover, the blood samples were inoculated into diphasic blood-culture bottles containing BHI broth and agar (Kusha Faravar Giti, Karaj, Iran). The samples were incubated at $37^{\circ} \mathrm{C}$ for at least 2 weeks. The remainder of the kidney samples and additional blood samples were used for microculture assay. Furthermore, control kidney and blood samples were also collected from uninfected mice for analysis.

\section{Microculture Assay}

For the assay, $50 \mu \mathrm{l}$ of blood was sampled using a sterile nonheparinized $1 \times 75 \mathrm{~mm}$ glass capillary tube. Blood sampling was performed by extracting the blood directly into the capillary tubes. For tissue samples, 20-50 mg of minced kidney was inserted into a sterile glass Pasteur pipette $(146 \times 6.5 \mathrm{~mm})$. The capillary tubes and Pasteur pipette were then loaded with 50-70 $\mu \mathrm{l}$ and approximately $200 \mu \mathrm{l}$ of RPMI 1640 medium (Sigma, St. Louis, MO, United States), respectively. The tubes were sealed with wax and incubated at $35^{\circ} \mathrm{C}$. One capillary tube sample and one Pasteur pipette sample were prepared for each animal. All samples were examined daily using an inverted microscope (Motic AE31 Elite Inverted Phase Contrast Microscope, magnification $100 \times$ ). Culture-negative samples were monitored for up to 30 days. Capillary tubes were examined under a light microscope (Nikon YS100 Biological Microscope, magnification $400 \times$ ). For the light microscopy analysis, two capillary tubes were placed horizontally on a microscope slide, and another slide was placed over them. The gap between the slides was filled with sterile water.

\section{Statistical Analysis}

Median survival time was estimated by the Kaplan-Meier method, compared among groups by the log-rank test. Tissue burden data of tested organ in the different experimental groups were analyzed by using the Kruskal-Wallis test in SPSS (version 17.0 for Windows; Chicago, IL, United States) and plotted using GraphPad Prism version 6.01 (Graph Prism Software Inc., United States). Categorical differences between positive and negative results for samples between the traditional culture (SDA) and microculture techniques were also determined by the equality of mean differences using a Chi-square $2 \times 2$ contingency table at a 95\% confidence interval. $p$-values $<0.05$ were considered as statistically significant.

\section{RESULTS}

An overall schematic of this study of the evaluation of microculture for the early diagnosis of mucormycosis in an immunocompetent murine model of disseminated infection compared with routinely performed methods is shown in Figure 1.

Preliminary experiments using three different inocula demonstrated that median survival time was 8,7 , and 5 days for mouse inoculation with $1 \times 10^{4}, 1 \times 10^{5}$, and $1 \times 10^{6} \mathrm{CFU} / \mathrm{mouse}$, respectively (Figure 2). The fungal tissue burden results are summarized in Figure 3. Fungal burden was significantly higher in mice infected with $1 \times 10^{6}$ CFUs compared to the other inocula levels in the $1 \times 10^{4}$ and $1 \times 10^{5} \mathrm{CFU} /$ mouse groups $(p<0.001)$.

Histopathological examination of kidney sections stained with PAS revealed irregular, broad, and non-septate hyphae, hallmarks of Mucorales, with several foreign bodies and langhans giant cells surrounded by a dense inflammatory response (Figure 4A). Subsequently, the performance of the microculture assay was evaluated by comparing it with culturing on media plates.

\section{Diagnostic Performance of the Microculture Assay Blood Samples}

The animals were euthanized on days 3-7 post-infection. Of the 90 blood samples, $26(28.9 \%)$ were positive using the microculture assay (Figures $4 \mathbf{B}, \mathbf{C}$ ). This included 6 in the lower inoculum group, 11 in the medium inoculum group, and 9 in the high inoculum group. Microculture samples in each group were positive between days 3 and 7 post-inoculations. In contrast, all blood samples were negative by culture (on SDA and BHI).

\section{Kidney Samples}

Microculture results were positive in 89 of 90 kidney samples (98.9\%). The overall positive conventional culture rate for the mouse kidney samples was 31.1\% (28/90). Therefore, 89 samples presented a positive result for kidney microculture assay, of which 28 were in accordance with the results of the SDA culture. There was significant difference in the positive microculture compared with SDA plates $(p<0.0001)$. All the samples collected from the 15 uninfected mice were negative by each assay. The concordance of detection (calculated sensitivity) for kidney microculture was 98.8\% (95\% CI 96.6-100), with a calculated assay specificity of $100 \%$. For kidney samples, the SDA culture presented the lower sensitivity (33.7\%; 95\% CI 23.9-43.5). These data demonstrate that the microculture assay ( $98.9 \%$ positivity) is superior to conventional culture ( $31.1 \%$ positivity) in this murine model in detecting $R$. arrhizus directly from the primary blood and kidney samples within 18-24 h of sampling.

\section{DISCUSSION}

In this study, we present the results of a microculture assay for timely culture of $R$. arrhizus. This is the first study to demonstrate that a microculture approach can be used for fungal culture within $24 \mathrm{~h}$ of sampling. Early diagnosis via multiple approaches is an important aspect of care in patients with mucormycosis (Blyth et al., 2011; Walsh et al., 2014; Hoenigl et al., 2018). A timely and efficient diagnosis, as well as an 


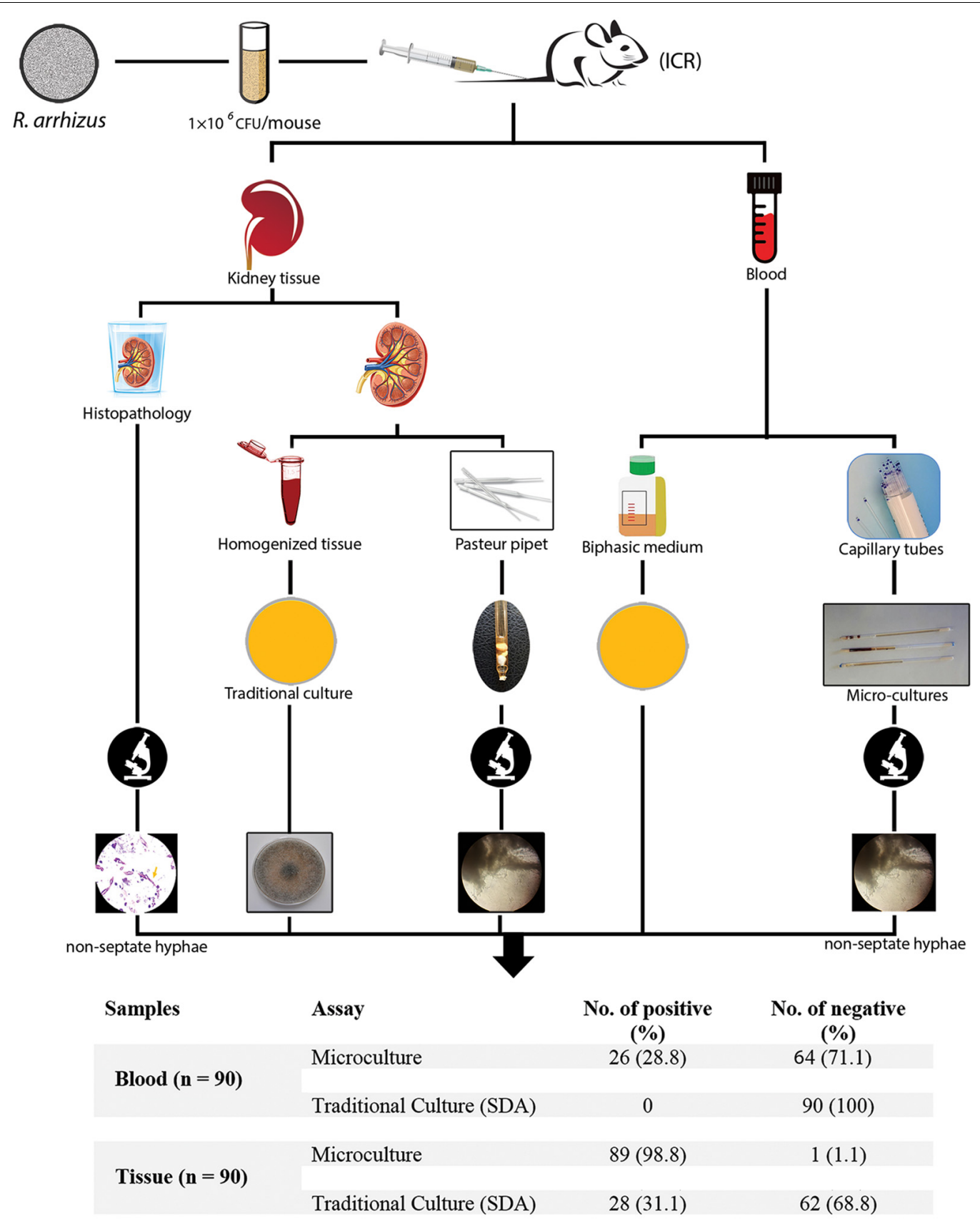

FIGURE 1 | Schematic of this study evaluating microculture compared with the traditional diagnostic methods.

aggressive multimodal treatment approach, is critical in the management of this fulminant progressive and invasive disease, as delays may result in an increased mortality risk (Walsh et al., 2014; Jeong et al., 2016). Indeed, a delay of more than 5 days of an effective antifungal therapy in patients with hematological malignancies leads to approximately twofold increase in 12 week mortality (Spellberg et al., 2005; Chamilos et al., 2008; Palejwala et al., 2016). Rapid mycological diagnostic methods may assist with timely initiation of appropriate antifungal therapy, which may prevent progressive tissue invasion, lead to decreased mortality, and overall improvement in healthcare utilization (i.e., shorter hospitalization duration and reduced costs). Histological analysis is an important diagnostic tool in the early management of this devastating disease (Skiada et al., 2018); however, the 24-h turnaround time of the microculture approach is considerably shorter than that of histological analysis (48$72 \mathrm{~h}$ ) (Dekio et al., 2015) or conventional culturing (3-7 days) (Skiada et al., 2018).

Conventional tissue fungal cultures are typically positive in only 50\% of mucormycosis cases (Skiada et al., 2018). Positive 


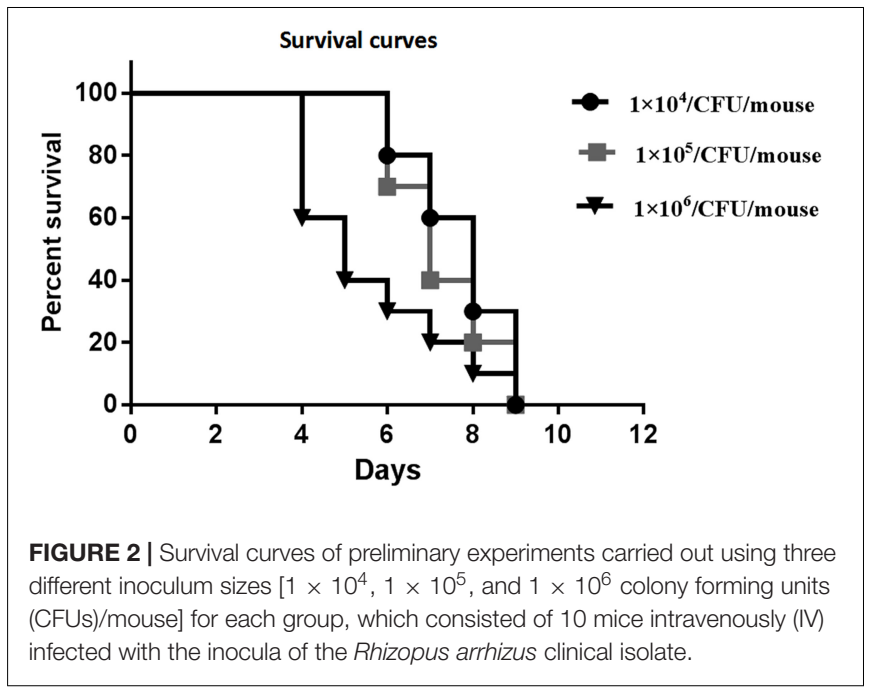

cultures and fungal identification, even at the genus level only, allow for the appropriate choice of antifungal regimens and further assessment of antifungal resistance patterns and emerging resistance (Cornely et al., 2014; Beardsley et al., 2018). Although some molecular identification methods may be able to provide results within a few hours, the microculture assay described in this study does not require specialized training or equipment. Surprisingly, in the present study, 26 of 90 blood samples were positive by microculture, while all blood samples were negative with traditional culture. Increased $\mathrm{CO}_{2}$ levels during incubation, leading to a lower $\mathrm{pH}$, may facilitate the growth of $R$. arrhizus in microculture tubes.

Culture-based methods for fungal identification are generally practical, economical, and accessible. The microculture method presented in this study is relatively rapid and easy to perform. Hence, this approach could also be considered for the diagnosis of other fungal infections, which may be challenging using traditional culture methods.

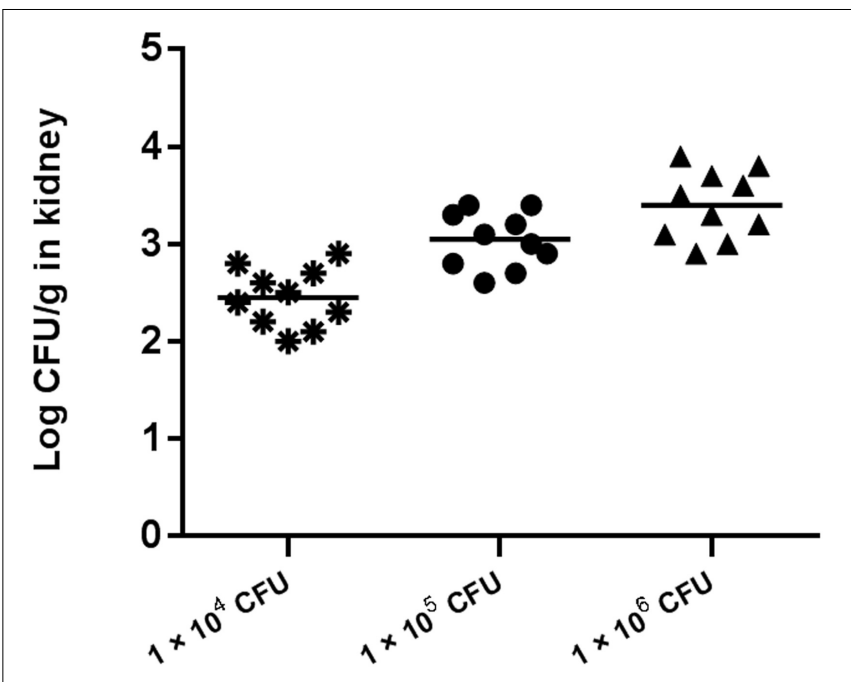

FIGURE 3 | Fungal tissue burden results in ICR mice infected with inocula of $1 \times 10^{4}, 1 \times 10^{5}$, and $1 \times 10^{6} \mathrm{CFU} /$ mouse on day 4 .

The promising results of the present study require confirmation through further studies. Microculture methods should be further assessed for the detection of other members of the order Mucorales as well as in other fungi. In addition, the performance of this assay in other murine models (e.g., pulmonary mucormycosis and in immunosuppressed hosts) should be evaluated. These additional studies are warranted given the relative ease of use of this method and the impact it may have within the clinical microbiology laboratory.

\section{AUTHOR'S NOTE}

A part of this work was presented as a poster presentation at the 20th Congress of the International Society for Human and Animal Mycology (ISHAM), Amsterdam, 30 June to 4 July 2018.

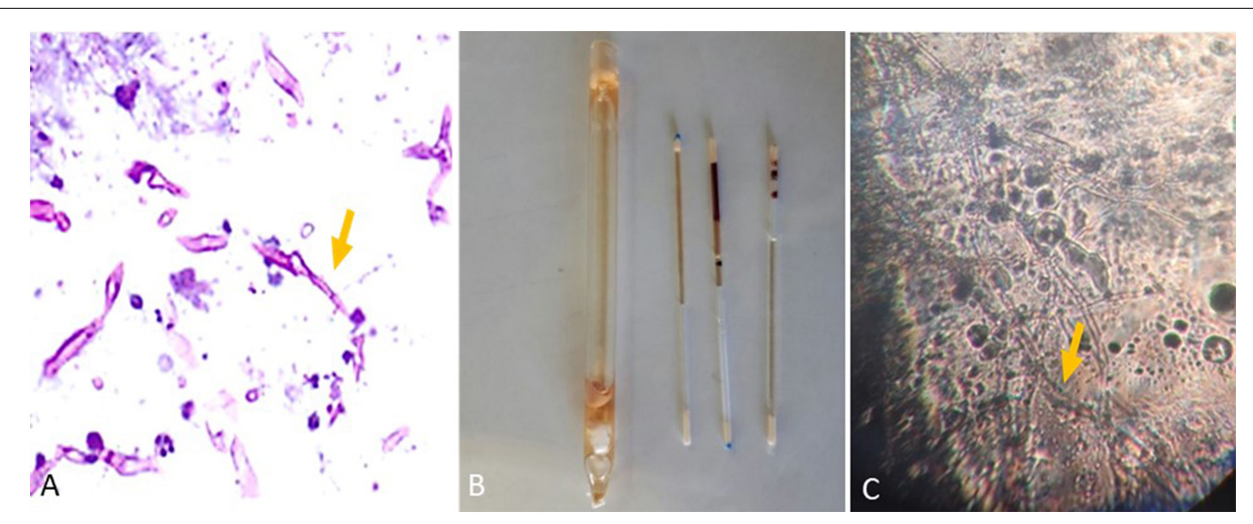

FIGURE 4 | (A) Representative histopathological periodic acid-Schiff (PAS) section of kidney from Institute of Cancer Research (ICR) female mice intravenously infected with $R$. arrhizus $\left(1 \times 10^{6} \mathrm{CFU} /\right.$ mouse) tested on day 7 post-challenge. (B) Blood and tissue sampling for the microculture assay using a sterile non-heparinized glass capillary tube. (C) Images captured $18 \mathrm{~h}$ after commencing the experiment (Mucorales hyphae). 


\section{DATA AVAILABILITY STATEMENT}

The raw data supporting the conclusions of this article will be made available by the authors, without undue reservation, to any qualified researcher.

\section{ETHICS STATEMENT}

This study was carried out in accordance with the recommendations of Guide for the Care and Use of Laboratory Animals, Committee for the Update of the Guide for the Care and Use of Laboratory Animals. The protocol was approved by the Ethics and Research Committee of Mazandaran University of Medical Sciences, Sari, Iran (IR.MAZUMS.REC.1397.9).

\section{AUTHOR CONTRIBUTIONS}

$\mathrm{AV}$ and $\mathrm{HB}$ contributed to the design and implementation of the research, and drafted the manuscript. $\mathrm{AV}, \mathrm{HB}$, and $\mathrm{HF}$ curated the data. AV, $\mathrm{HB}$, and VA performed the formal analysis of

\section{REFERENCES}

Beardsley, J., Halliday, C. L., Chen, S. C., and Sorrell, T. C. (2018). Responding to the emergence of antifungal drug resistance: perspectives from the bench and the bedside. Future Microbiol. 13, 1175-1191. doi: 10.2217/fmb-2018-0059

Ben-Ami, R., Luna, M., Lewis, R. E., Walsh, T. J., and Kontoyiannis, D. P. (2009). A clinicopathological study of pulmonary mucormycosis in cancer patients: extensive angioinvasion but limited inflammatory response. J. Infect. 59, 134-138. doi: 10.1016/j.jinf.2009.06.002

Blyth, C. C., Gomes, L., Sorrell, T. C., da Cruz, M., Sud, A., and Chen, S. C. (2011). Skull-base osteomyelitis: fungal vs. bacterial infection. Clin. Microbiol. Infect. 17, 306-311. doi: 10.1111/j.1469-0691.2010.03231.x

Chakrabarti, A., and Singh, R. (2014). Mucormycosis in India: unique features. Mycoses 57(Suppl. 3), 85-90. doi: 10.1111/myc.12243

Chamilos, G., Lewis, R. E., and Kontoyiannis, D. P. (2008). Delaying amphotericin B-based frontline therapy significantly increases mortality among patients with hematologic malignancy who have zygomycosis. Clin. Infect. Dis. 47, 503-509. doi: $10.1086 / 590004$

Conti, H. R., Huppler, A. R., Whibley, N., and Gaffen, S. L. (2014). Animal models for candidiasis. Curr. Protoc. Immunol. 105, 11-17. doi: 10.1002/0471142735. im1906s105

Cornely, O. A., Alastruey-Izquierdo, A., Arenz, D., Chen, S. C. A., Dannaoui, E., Hochhegger, B., et al. (2019). Global guideline for the diagnosis and management of mucormycosis: an initiative of the European confederation of medical mycology in cooperation with the mycoses study group education and research consortium. Lancet Infect. Dis. 19, e405-e421. doi: 10.1016/S14733099(19)30312-3

Cornely, O. A., Arikan-Akdagli, S., Dannaoui, E., Groll, A. H., Lagrou, K., Chakrabarti, A., et al. (2014). European society of clinical microbiology and infectious diseases fungal infection study group; European confederation of medical mycology. ESCMID and ECMM joint clinical guidelines for the diagnosis and management of mucormycosis 2013. Clin. Microbiol. Infect. 20(Suppl. 3), 5-26. doi: 10.1111/1469-0691.12371

Dadwal, S. S., and Kontoyiannis, D. P. (2018). Recent advances in the molecular diagnosis of mucormycosis. Expert Rev. Mol. Diagn. 18, 845-854. doi: 10.1080/ 14737159.2018.1522250

Danion, F., Aguilar, C., Catherinot, E., Alanio, A., DeWolf, S., Lortholary, O., et al. (2015). Mucormycosis: new developments into a persistently devastating infection. Semin. Respir. Crit. Care Med. 36, 692-705. doi: 10.1055/s-00351562896 the study and contributed to funding acquisition and project administration. AV, MF, HF, and LF provided the methodology for this study. MI and NW validated the data and revised the manuscript. All authors contributed to approve the final version of the manuscript.

\section{FUNDING}

The work of HB partially was supported by a grant (Nr. 9) from the School of Medicine, Mazandaran University of Medical Sciences, Sari, Iran, which we gratefully acknowledge.

\section{ACKNOWLEDGMENTS}

The authors are indebted to Dr. Sarvi and his colleagues for their excellent technical assistance with the animal experiments at the Mazandaran University of Medical Sciences, Sari, Iran. In addition, they would like to acknowledge Hossein Chehre for providing the photographic plates.

Dekio, F., Bhatti, T. R., Zhang, S. X., and Sullivan, K. V. (2015). Positive impact of fungal histopathology on immunocompromised pediatric patients with histology-proven invasive fungal infection. Am. J. Clin. Pathol. 144, 61-67. doi: 10.1309/AJCPEMVYT88AVFKG

Farmakiotis, D., and Kontoyiannis, D. P. (2016). Mucormycoses. Infect. Dis. Clin. North Am. 30, 143-163. doi: 10.1016/j.idc.2015.10.011

Hammond, S. P., Bialek, R., Milner, D. A., Petschnigg, E. M., Baden, L. R., and Marty, F. M. (2011). Molecular methods to improve diagnosis and identification of mucormycosis. J. Clin. Microbiol. 49, 2151-2153. doi: 10.1128/JCM.0025611

Hoenigl, M., Gangneuxm, J. P., Segal, E., Alanio, A., Chakrabarti, A., Chen, S. C., et al. (2018). Global guidelines and initiatives from the European confederation of medical mycology to improve patient care and research worldwide: new leadership is about working together. Mycoses 61, 885-894. doi: 10.1111/myc. 12836

Jeong, S. J., Lee, J. U., Song, Y. G., Lee, K. H., and Lee, M. J. (2016). Delaying diagnostic procedure significantly increases mortality in patients with invasive mucormycosis. Mycoses 58, 746-752. doi: 10.1111/myc. 12428

Katragkou, A., Walsh, T. J., and Roilides, E. (2014). Why is mucormycosis more difficult to cure than more common mycoses? Clin. Microbiol. Infect. 20(Suppl. 6), 74-81. doi: 10.1111/1469-0691.12466

Kontoyiannis, D. P., and Lewis, R. E. (2011). How I treat mucormycosis. Blood 118, 1216-1224. doi: 10.1182/blood-2011-03-316430

Lewis, R. E., Cahyame-Zuniga, L., Leventakos, K., Chamilos, G., Ben-Ami, R., Tamboli, P., et al. (2013). Epidemiology and sites of involvement of invasive fungal infections in patients with haematological malignancies: a 20-year autopsy study. Mycoses 56, 638-645. doi: 10.1111/myc.12081

Nagao, K., Ota, T., Tanikawa, A., Takae, Y., Mori, T., Udagawa, S., et al. (2005). Genetic identification and detection of human pathogenic Rhizopus species, a major mucormycosis agent, by multiplex PCR based on internal transcribed spacer region of rRNA gene. J. Dermatol. Sci. 39, 23-31. doi: 10.1016/j.jdermsci. 2005.01.010

National Research Council (2011). Guide for the Care and Use of Laboratory Animals, 8th Edn. Washington, DC: National Academies Press.

Palejwala, S. K., Zangeneh, T. T., Goldstein, S. A., and Lemole, G. M. (2016). An aggressive multidisciplinary approach reduces mortality in rhinocerebral mucormycosis. Surg. Neurol. Int. 7:61. doi: 10.4103/2152-7806.182964

Petrikkos, G., Skiada, A., Lortholary, O., Roilides, E., Walsh, T. J., and Kontoyiannis, D. P. (2012). Epidemiology and clinical manifestations of mucormycosis. Clin. Infect. Dis. 54(Suppl. 1), S23-S34. doi: 10.1093/cid/cir866 
Pilmis, B., Alanio, A., Lortholary, O., and Lanternier, F. (2018). Recent advances in the understanding and management of mucormycosis. F1000Res 7:F1000FacultyRev-1429. doi: 10.12688/f1000research.15081.1

Prakash, H., and Chakrabarti, A. (2019). Global epidemiology of mucormycosis. J. Fungi. 5:26. doi: 10.3390/jof5010026

Rickerts, V. (2016). Identification of fungal pathogens in Formalin-fixed, Paraffinembedded tissue samples by molecular methods. Fungal Biol. 120, 279-287. doi: 10.1016/j.funbio.2015.07.002

Skiada, A., Lass-Floerl, C., Klimko, N., Ibrahim, A., Roilides, E., and Petrikkos, G. (2018). Challenges in the diagnosis and treatment of mucormycosis. Med. Mycol. 56(Suppl. 1), 93-101. doi: 10.1093/mmy/ myx101

Skiada, A., Rigopoulos, D., Larios, G., Petrikkos, G., and Katsambas, A. (2012). Global epidemiology of cutaneous zygomycosis. Clin. Dermatol. 30, 628-632. doi: 10.1016/j.clindermatol.2012.01.010

Spellberg, B., Edwards, J. Jr., and Ibrahim, A. (2005). Novel perspectives on mucormycosis: pathophysiology, presentation, and management. Clin. Microbiol. Rev. 18, 556-569. doi: 10.1128/CMR.18.3.556-569.2005

Vaezi, A., Moazeni, M., Rahimi, M. T., de Hoog, S., and Badali, H. (2016). Mucormycosis in Iran: a systematic review. Mycoses 59, 402-415. doi: 10.1111/ myc. 12474
Walsh, T. J., Skiada, A., Cornely, O. A., Roilides, E., Ibrahim, A., Zaoutis, T., et al. (2014). Development of new strategies for early diagnosis of mucormycosis from bench to bedside. Mycoses 57(Suppl. 3), 2-7. doi: 10.1111/myc.12249

Yamazaki, T., Kume, H., Murase, S., Yamashita, E., and Arisawa, M. (1999). Epidemiology of visceral mycoses: analysis of data in annual of the pathological autopsy cases in Japan. J. Clin. Microbiol. 37, 1732-1738.

Conflict of Interest: The authors declare that the research was conducted in the absence of any commercial or financial relationships that could be construed as a potential conflict of interest.

The reviewer AA-H declared past co-authorship with several of the authors, AV, $\mathrm{HF}, \mathrm{MI}$, and $\mathrm{HB}$, to the handling Editor.

Copyright (C) 2020 Vaezi, Fakhim, Ilkit, Faeli, Fakhar, Alinejad, Wiederhold and Badali. This is an open-access article distributed under the terms of the Creative Commons Attribution License (CC BY). The use, distribution or reproduction in other forums is permitted, provided the original author(s) and the copyright owner(s) are credited and that the original publication in this journal is cited, in accordance with accepted academic practice. No use, distribution or reproduction is permitted which does not comply with these terms. 